\title{
Go with the flow-clinical importance of flow curves during mechanical ventilation: A narrative review
}

\author{
Natsumi T. Hamahata, MD¹, Ryota Sato, MD¹, Ehab G. Daoud, MD, FACP, FCCP ${ }^{1,2,3}$
}

\begin{abstract}
NT Hamahata, RS Sato, EG Daoud. Go with the flow-clinical importance of flow curves during mechanical ventilation: A narrative review. Can J Respir Ther 2020;56:11-20. doi: 10.29390/cjrt-2020-002.

Most clinicians pay attention to tidal volume and airway pressures and their curves during mechanical ventilation. On the other hand, inspiratory-expiratory flow curves also provide a plethora of information, but much less attention is paid to them. Flow curves chronologically show the velocity and direction of inspiration and expiration and are influenced by the respiratory mechanics, the patient's effort, and the mode of ventilation and its settings. When the ventilator setting does not synchronize with the patient's respiratory pattern, the patient can easily have worsening breathing effort, patient-ventilator asynchrony, which can lead to prolonged ventilator support or lung injury. The information provided by the flow curves during mechanical ventilation, such as respiratory mechanics, the patient's effort, and patient-ventilator interactions, are very helpful when adjusting the ventilator setting. If clinicians can monitor and assess the flow curves information appropriately, it can be a useful diagnostic and therapeutic tool at the bedside. There may be association between inspiratory effort and flow, and this may further guide us, especially in the weaning process and when patients are not synchronizing with the ventilator. In this review, we try to gather information about "flow" that is scattered around in the literature and textbooks in one place. We will summarize the different flow waveforms utilized in commonly used ventilator modes with their advantages and disadvantages, information gained by the flow curves (i.e., flow-time, flow-volume, and flow-pressure), how to detect and manage asynchronies, and some ideas for future uses. Flow waveforms shapes and patterns are very beneficial for the management of patients undergoing mechanical ventilatory support. Attention to those waveforms can potentially improve patient outcomes. Clinicians should be familiar with this information and how to act upon them.
\end{abstract}

Key Words: flow curve, mechanical ventilation, ventilator synchrony, respiratory mechanics, ventilator weaning

\section{INTRODUCTION}

Measurements of flow, volumes, and pressures are of paramount importance during mechanical ventilation. New generation ventilators use different flow and pressure sensors for adequate pressure and gas flow to and from the ventilator to the patient. The flow sensors not only measure flow but they also act as trigger sensors for the patient's spontaneous breathing, integrate the flow signals to tidal volume, and send feedback for ventilator adjustments in closed-loop systems [1].

Most ventilators display the graphics of flow $(\mathrm{L} / \mathrm{min})$, pressure $\left(\mathrm{cmH}_{2} \mathrm{O}\right)$, and tidal volume $(\mathrm{mL})$ on the Y-axis against time (seconds) on the $\mathrm{X}^{2}$-axis (Figure 1). They also can display those graphics as loops against each other (e.g., pressure-volume, flow-volume, and flow-pressure.) Different ventilator manufacturers use different commercial flow sensors and place those sensors either proximal to the patient or to the ventilator. The disequilibrium between the tidal volume delivered to the patient and the one measured inside the ventilator can occur due to the pneumatic behavior of the breathing circuit compliance. This is flow-rate dependent, and a previous study investigating the use of pneumotachometer in ventilated infants showed a difference between expiratory tidal volume measured by ventilator and that measured with pneumotachometer at the airway or inside the trachea [2]. However, a pneumotachometer is not routinely used and some equations were developed to compensate for the circuit compliance. For these reasons, flow and subsequently tidal volume can be different from one ventilator to another even in the same patient or same respiratory mechanics [3, 4].

\section{Expiratory flow}

Expiration is usually a passive process due to the elastic recoil of the respiratory system (both lungs and chest wall) by the kinetic energy stored during inspiration, though it might not be the case in some patientventilator asynchronies. Expiratory flow is dependent on the applied airway pressure (driving pressure) and tidal volume. The peak expiratory flow (PEF) and expiratory flow time are dependent on the time constant (TC) that results in the Resistance X Compliance of the respiratory system. At each TC, the flow is estimated to decay to about $37.8 \%$ from its previous value [5]. At least four TCs are required for the lung to reach the resting functional residual capacity (FRC) before the next breath $[5,6]$. As the compliance and resistance become higher (e.g., cases of chronic obstructive airway disease (COPD)), the PEF is reduced and the expiratory TC is prolonged. If this expiratory time is not long enough for the new breath to start after expiration reaches baseline, hyperinflation (auto-positive endexpiratory pressure [PEEP]) will develop [7]. In addition to its well-known adverse hemodynamic affect, auto-PEEP worsens oxygenation and ventilation by increasing the dead space and work of breathing (WOB). The dead space causes ventilation-perfusion mismatch and with increased WOB will cause more oxygen consumption and $\mathrm{CO}_{2}$ production [8]. Additionally, it places the diaphragm at a disadvantage causing diaphragmatic weakness, patient-ventilator asynchrony, or the inability to trigger the ventilator [9].

On the other hand, restrictive lung disease has very low TC, thus the $\mathrm{PEF}$ is high and the lungs tends to return to FRC rapidly [5].

A recent condition termed the expiratory flow limitation (EFL) is the inability of the expiratory flow to increase despite an increase in the expiratory driving pressure (the pressure difference between the alveoli and the airway opening during expiration) [10]. The exact mechanisms and anatomical location are not entirely clear but the most commonly cited mechanism is dynamic airway compression that occurs when the intraluminal pressure equals the pressure in the surrounding structure (pleural space) and there is

\footnotetext{
${ }^{1}$ Department of Internal Medicine, John A. Burns School of Medicine, University of Hawaii at Manoa, Honolulu, HI, USA

${ }^{2}$ Respiratory Care Program, Kapiolani Community College, Honolulu, HI, USA

${ }^{3}$ Critical Care Department, Kuakini Medical Center, Honolulu, HI, USA

Correspondence: Natsumi T. Hamahata, 1356 Lusitana St, UH tower $7^{\text {th }}$ floor, Honolulu HI, 96817 USA; Tel: (808)-492-8329; Fax: (808)-586-7486.

Email:natsumi.tanabe.md@gmail.com
}

Published online at https://www.cjrt.ca on 22 July 2020 


\section{FIGURE 1.}

Basic waveform for PCV mode and VCV mode. (A) Example of PCV mode using decelerating inspiratory flow wave. (B) Example of VCV mode using a constant (square) inspiratory flow with an inspiratory pause. Top yellow curve is airway pressure in $\mathrm{cmH}_{2} \mathrm{O}$, middle pink curve is flow in L/min, bottom green is tidal volume in $\mathrm{mL}$. $A$ is peak inspiratory pressure (PIP), $B$ is plateau pressure ( $P$ plat) after inspiratory pause in VCV (notice that PIP and $P$ plat are the same in PCV), $C$ is expiratory pressure (PEEP), D is peak inspiratory flow (PIF) (i.e., the maximum positive flow during inspiration), $E$ is peak expiratory flow (PEF) (i.e., the maximum negative flow). Time zero to $B$ and $D$ to $E$ are the inspiratory time (I time), $C$ and $E$ to $F$ are expiratory time (E time).

A

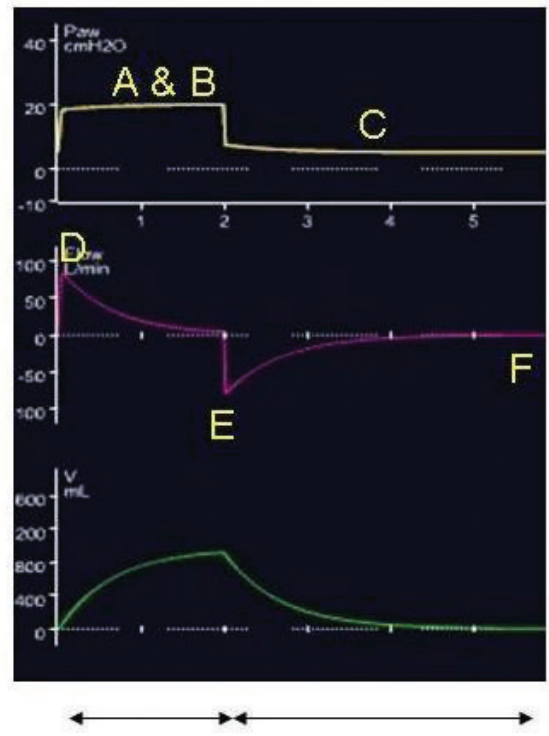

Inspiration

Expiration
B

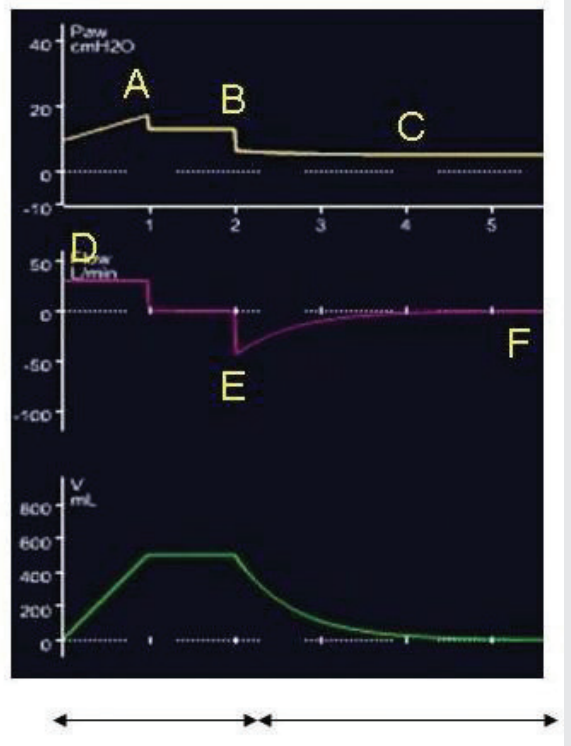

Inspiration

Expiration

Time in seconds

no pressure gradient. Dynamic collapse occurs from this point to the downstream segment. It is a relatively frequent phenomenon among critically ill patients, and usually goes unrecognized especially in COPD patients, but it can also occur in obesity, heat failure, and Acute Respiratory Distress Syndrome (ARDS) patients. One of the methods to diagnose EFL is to alter the expiratory driving pressure and compare the difference of flow in each condition at the same lung volume. This method requires the operator to manually reduce the PEEP while recording two consecutive flow-volume loops. Reducing the PEEP will increase the expiratory driving pressure, and this should generate higher expiratory flow if there is no EFL. Also, small airway collapse occurs during exhalation and can cause auto-PEEP. However, the auto-PEEP caused by EFL happens to be relatively refractory to changes in expiratory time compared with auto-PEEP generated by insufficient expiratory time and patients with EFL have greater auto-PEEP than patients without EFL. Applied PEEP can help improve such occurrences [10].

A new ventilator mode termed flow-controlled ventilation that uses linear inspiratory and expiratory flow in contrast to the exponential expiratory flow in the volume-and pressure-controlled modes has shown enhancement of lung aeration in the dependent lung region and consequently improved gas exchange and attenuation of lung injury in animal studies and may provide a novel option for lung-protective ventilation [9] (Figure 2).

Inspiratory flow

Types of inspiratory waveforms

Essentially there are five different inspiratory flow waveforms output from the ventilator depending on the mode used (Figure 3).

Most pressure-controlled ventilator (PCV) modes use the decelerating or descending waveform where the inspiratory flow rises fast and exponentially decays down during the inspiratory cycle. The pressure in the lung increases as it gets filled and pressure gradient diminishes, which drops the flow continuously during inspiration. The square or constant waveform is used mainly on the volume-controlled ventilator (VCV) modes, the flow value quickly rises linearly to the value set on the ventilator and then remains constant during inspiration until the tidal volume $\left(\mathrm{V}_{\mathrm{T}}\right)$ has been delivered then falls rapidly before exhalation begins. Most new generation ventilators can apply the descending waveform in the VCV mode as well to reduce the peak inspiratory pressure [11, 12], which indicates that the influence of the airway resistance is minimized in the descending waveform. A decelerating waveform was also reported to reduce the dead space, the alveolar-arterial gradient and the WOB $[13,14]$. The accelerating or ascending waveform starts with the low flow but steadily rises during inspiration, this has been eliminated from most new ventilators for its unfavorable effects on the patient's WOB and asynchronies. It is rare but some ventilators still use the sinusoidal waveform that reciprocates the normal spontaneous breathing with an increase followed by a decrease during the inspiration.

The type of waveform used can affect the peak inspiratory airway pressure (PIP) [11], mean airway pressure (Paw), and inspiratory time (I time) [15], which can subsequently affect ventilation and oxygenation. We will focus on the decelerating and constant waveforms as they are the most commonly used by virtually all modes of mechanical ventilation.

Factors affecting waveforms

Multiple factors affect the inspiratory flow shape, peak inspiratory flow (PIF), and flow (inspiratory) time. Particularly, the inspiratory time, respiratory rate $(\mathrm{RR})$, respiratory system mechanics (compliance, resistance, and TC), rise time, and patients' muscle efforts (Pmus). 


\section{FIGURE 2.}

Flow-controlled ventilation. Top panel shows is airway pressure, bottom panel shows the linear inspiratory and expiratory flow curves characteristic of this mode.
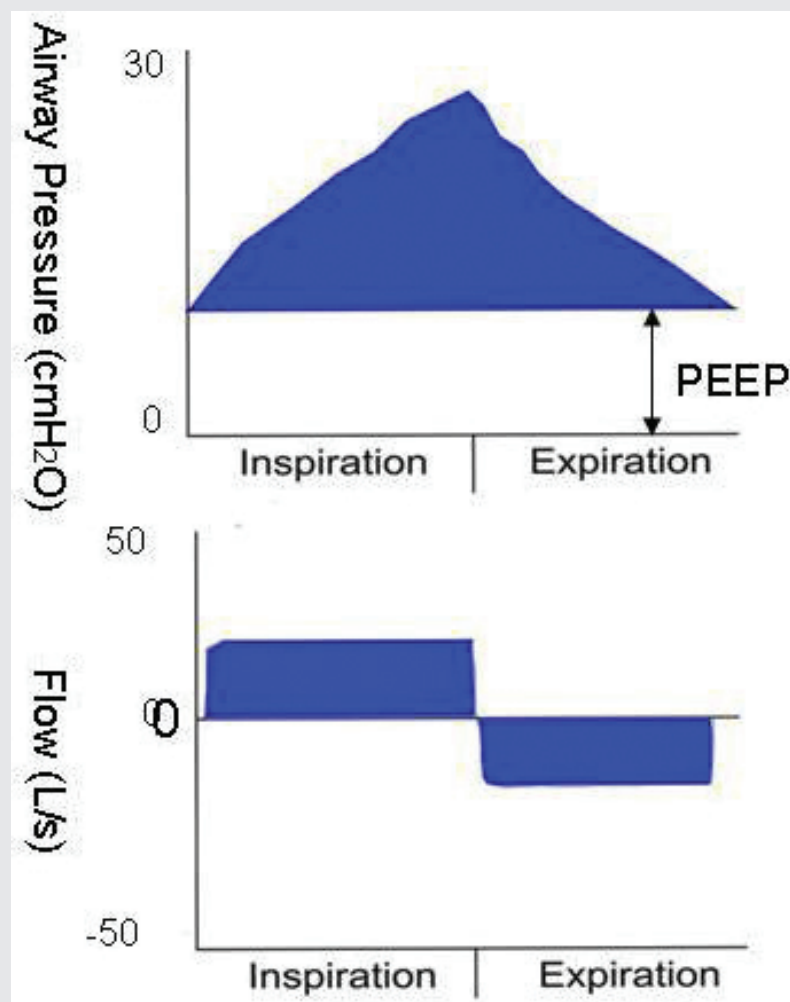

Time (seconds)

In the VCV modes using any flow waveforms, with a fixed flow rate, the PIF will remain constant while the inspiratory time will be variable according to the flow rate (higher with lower flow and vice versa), and the peak inspiratory pressure (PIP) will vary according to the respiratory mechanics (higher with high resistance and low compliance). On the other hand, when the Inspiratory:Expiratory ratio is fixed, the PIF and inspiratory time will be variable according to the RR (i.e., the PIF increases and the inspiratory time shortens with the higher RR and vice versa). The PIP will change similar to the fixed flow rate (Figure 4).

In the PCV modes, flow is variable as the ventilator constantly adjusts the flow to maintain preset pressure and inspiratory time. The PIF is higher with low compliance (e.g., ARDS) and conversely lower with high resistance and compliance (e.g., COPD) (Figure 4). The PIF is will increase with a higher RR and vice versa.

The effect of patients' Pmus on flow is not very well studied or reported, but based on the patient's observation and simulator work, the higher Pmus, the higher the PIF and higher mean inspiratory flow [16] (Figure 5).

Effects of different flows on oxygenation, WOB, and outcomes

The effects of different inspiratory flow patterns on respiratory mechanics, oxygenation, ventilation, and WOB have been controversial and nonconclusive. Some studies showed improved oxygenation, respiratory mechanics, and dead space with the decelerating flow ramp, while others were not able to reproduce the same conclusions [11-13].
It is widely accepted that the decelerating waveforms can achieve higher mean airway pressures (surrogate for oxygenation) and alveolar recruitment with lower peak inspiratory flow, while a constant airflow ramp produces shorter inspiratory time with higher PIF [13]. Additionally, the decelerating waveform in PCV modes have been proposed to decrease WOB and patient-ventilator asynchrony due to the initial high flow in conditions of air hunger and due to the unrestricted flow according to patient's efforts and respiratory mechanics as mentioned above. On the other hand, patientventilator asynchronies may happen more frequently in the constant flow ramp as the patients are only allowed to inhale with the same flow (Figure 5).

When compared together, sinusoidal waveforms and constant square waveforms did not show a significant difference in WOB or inspiratory pressure [13].

Effects of flows on ventilator-induced lung injury (VILI)

VILI has been increasingly understood and is usually related to volutrauma, baro-trauma, atelect-trauma, and bio-trauma. The effect of high PIF was suggested to cause accelerated VILI in animal models using very high $\mathrm{V}_{\mathrm{T}}(20-30 \mathrm{~mL} / \mathrm{kg})$ [17]. The results of high PIF is unclear in human studies because it is unethical to prescribe such high tidal volumes.

\section{CLINICAL USE}

Calculating tidal volumes

Most modern ventilators do not have a spirometer built-in to measure inhaled and exhaled tidal volumes. However, the displayed tidal volumes are obtained from integration of the flow rate divided by time (i.e., flow/time).

\section{Trigger}

Breaths trigger variable refers to starting the breath during mechanical ventilation. It is usually either time triggered (based on set respiratory rate) or patient triggered. Patient-triggered breaths may be either flow or pressure triggered. For most patients, pressure sensitivity settings from $-0.5 \mathrm{cmH}_{2} \mathrm{O}$ to $-2.0 \mathrm{cmH}_{2} \mathrm{O}$ are safe and effective. With the flow trigger, the typical flow trigger setting is $1-3 \mathrm{~L} / \mathrm{min}$ [1]. Studies comparing pressure versus flow triggering have shown that flow triggering may be more comfortable than pressure-triggered breaths with resultant decreased WOB, though this has been challenged by some authors [18]. More recent studies did not show a significant difference in patient's responses with flow and pressure triggering during pressure support ventilation (PSV) [19].

In the presence of auto-PEEP, the patient's effort must overcome the whole amount of auto-PEEP before a pressure or flow change occurs at the proximal airway to trigger the ventilator. In this scenario, flow triggering showed superiority to pressure triggering [20]. Adjusting the trigger sensitivity or changing from the pressure to flow triggering may help with the trigger asynchrony. The neural trigger (diaphragmatic electromyogram) or the electrical activity of the diaphragm is not affected by auto-PEEP and therefore might be the most appropriate triggering mode for these patients.

\section{Cycle}

Breaths cycle variable refers to the end of inspiration and transitioning to expiration. The most common cycle variables are flow, time, or pressure. VCV modes usually use flow cycle where the breath ends after a set flow rate and tidal volume is applied. Pressure support mode is a common mode where the ventilator cycles when a predetermined percentage decay of the PIF is reached, it is usually called expiratory sensitivity or expiratory trigger sensitivity (historically around $25 \%$ but can be adjustable on most ventilators). Attention must be paid when adjusting this level as it can affect patients' effort and comfort, respiratory mechanics, and asynchronies. If it is set too low, the ventilator will continue inspiration even after the respiratory muscles have relaxed. If it is set too high, the ventilator will stop delivering air even if the respiratory muscles are 


\section{FIGURE 3.}

Typical flow waveforms used by different modes of ventilators. Upper yellow curves are the airway pressures in $\mathrm{cmH}_{2} \mathrm{O}$, middle pink curves are flows in L/min, and lower green curves are tidal volumes in $\mathrm{mL}$. (A) Constant or square flow waveform, (B) descending, (C) sinusoidal, (D) decelerating, and (E) ascending.
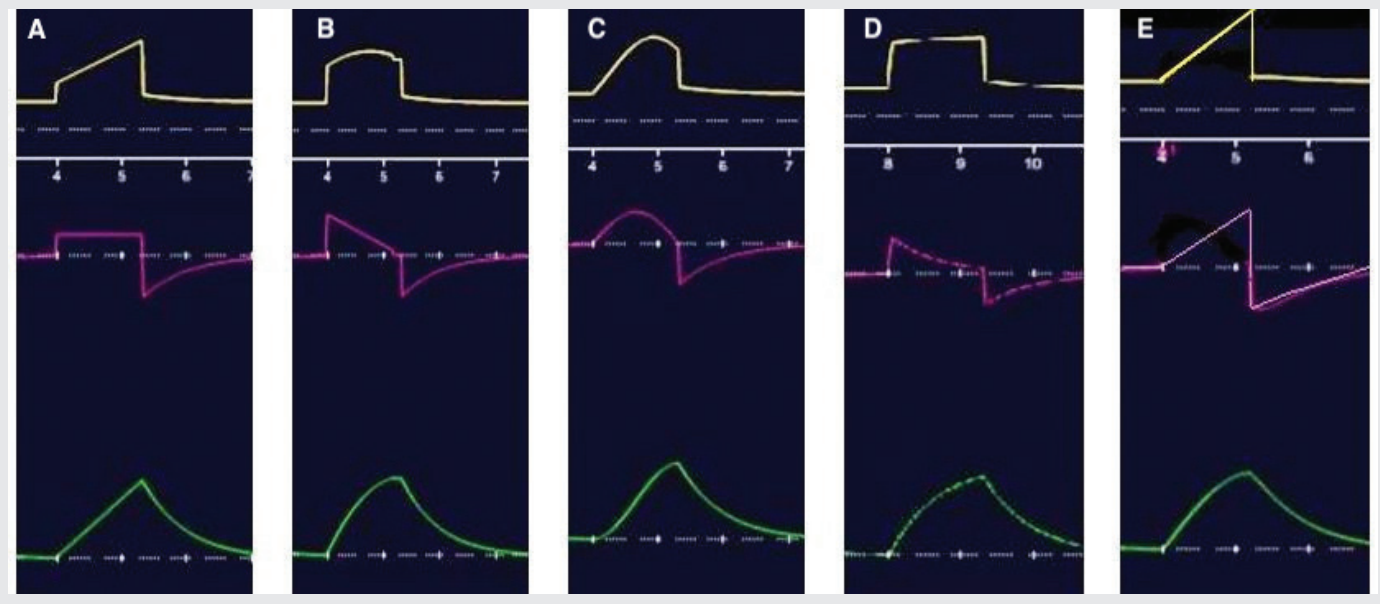

\section{FIGURE 4.}

Volume-controlled mode with constant square waveform in different clinical scenarios (A-C) and pressure-controlled mode showing different flow appearance in different clinical scenarios (D-F). (A) ARDS, (B) normal, (C) COPD showing missed effort (yellow arrow) and auto PEEP (red arrow), (D) normal resistance and compliance, (E) COPD with higher resistance and compliance, and (F) ARDS with normal resistance and low compliance. Upper yellow curves are the airway pressures in $\mathrm{cmH}_{2} \mathrm{O}$, middle pink curves are flows in L/min, and lower green curves are tidal volumes in $\mathrm{mL}$.
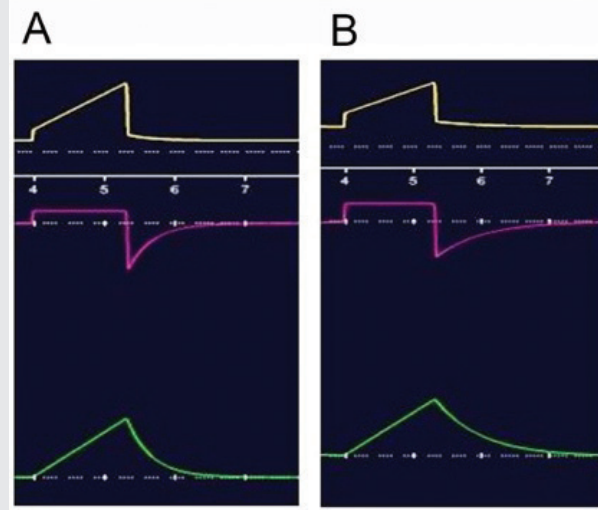

C
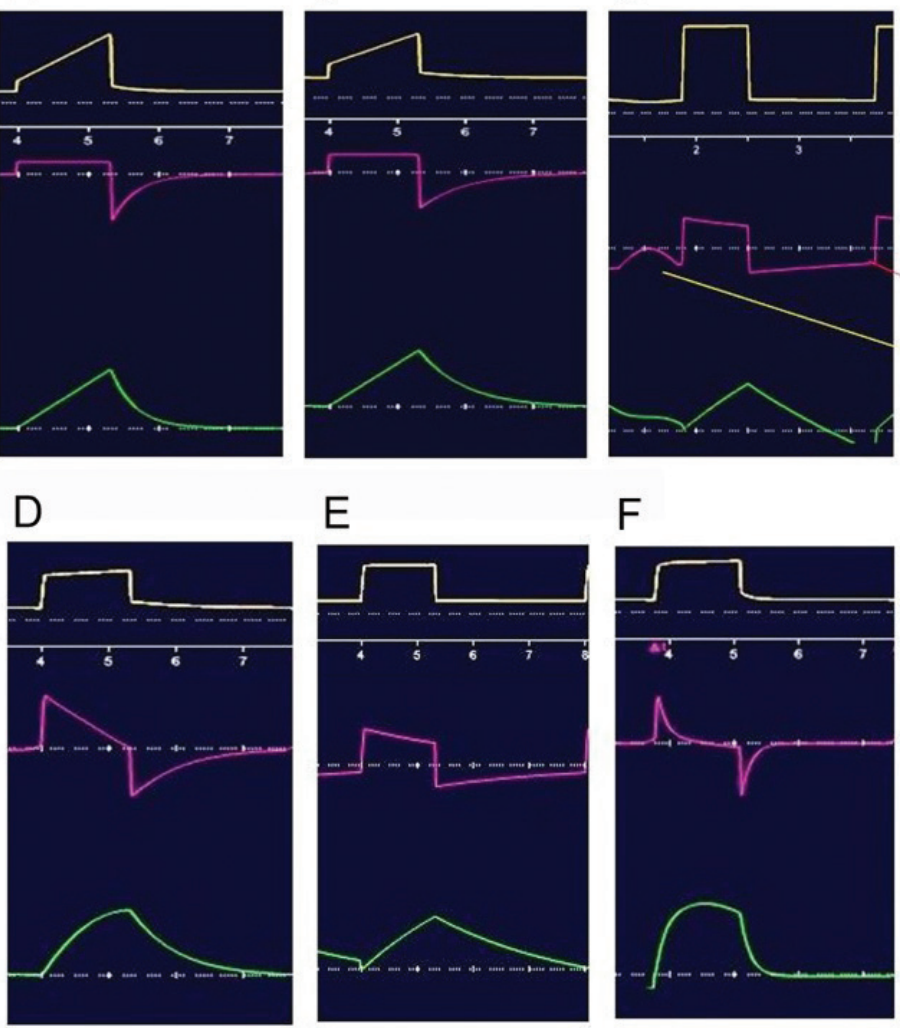

$\mathrm{F}$

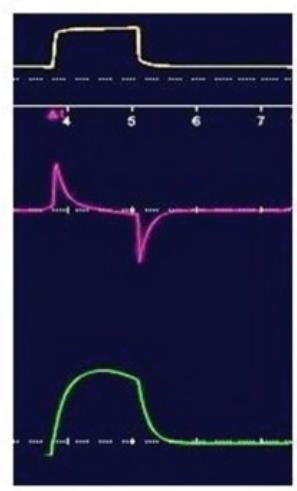




\section{FIGURE 5.}

Effect of inspiratory muscle pressure (Pmus) on shape of inspiratory flow (A-C) and effect of high inspiratory effort in patient ventilated with VC mode (D and E). (A) No spontaneous effort, (B) modest inspiratory effort, (C) high inspiratory effort. Note the increased upward concavity and increased PIF with the high effort, along the slight downward concavity on the airway pressure curve. (D) Patient with complete paralysis and no inspiratory effort, (E) patient with high inspiratory muscle effort, note the downward dipping in airway pressure (top yellow curve) but the unchanged fixed flow rate and PIF. Upper yellow curves are the airway pressures in $\mathrm{cmH}_{2} \mathrm{O}$, middle pink curves are flows in L/min, and lower green curves (for $\mathrm{A}-\mathrm{C}$ ) are tidal volumes in $\mathrm{mL}$.

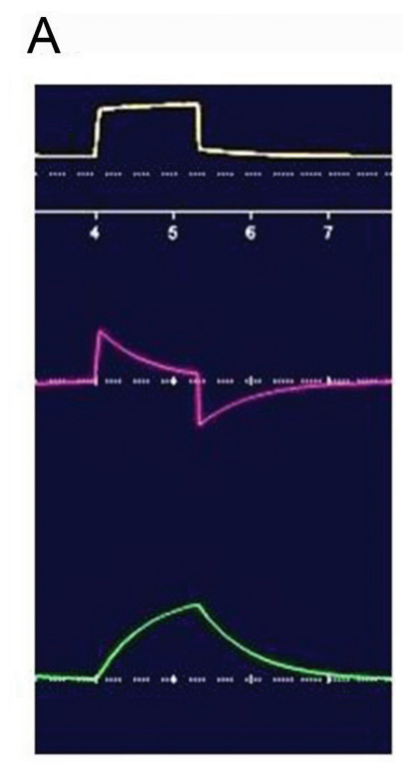

D

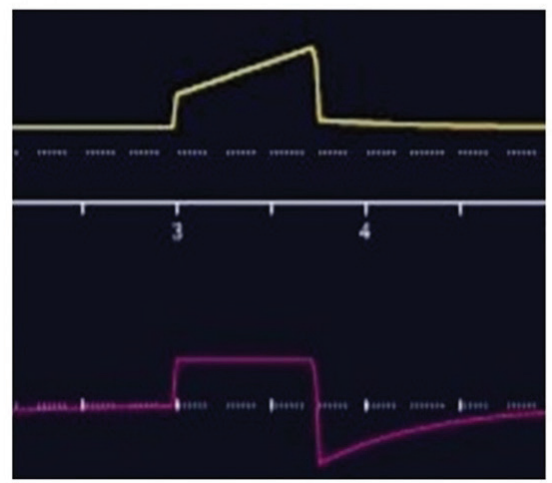

B

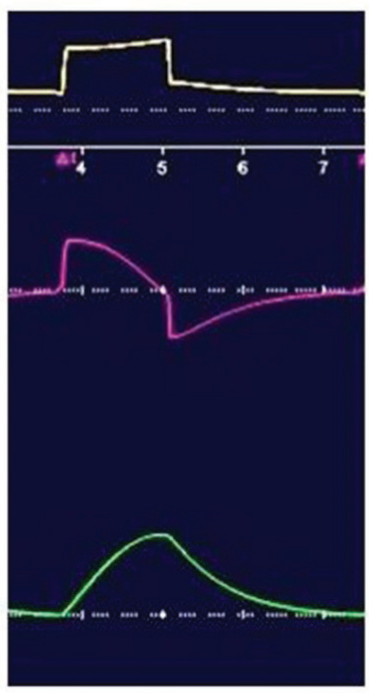

$\mathrm{E}$
C
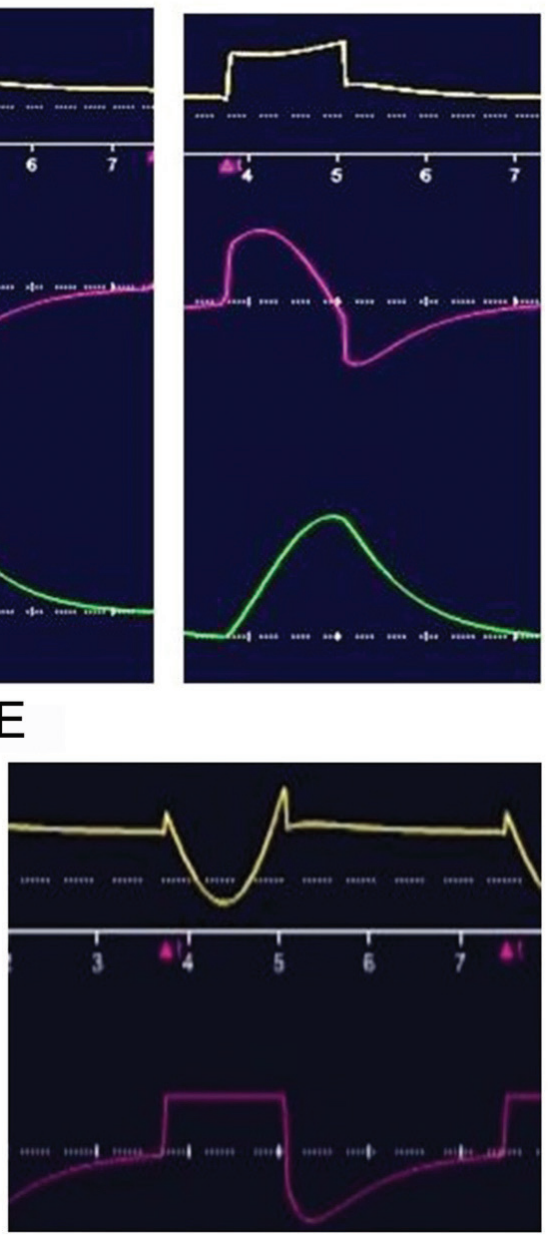

still contracted and can lead to double triggering, breath stacking, and lung injury (Figure 6).

Ideally, the ventilator should cycle to exhalation at the end of the neural inspiratory time. If breath terminates before the need for neural inhalation, the patient may double trigger the ventilator.

Automatic adjustment of ventilator settings in newer modes Adaptive support ventilation is one of the newer and intriguing modes of mechanical ventilation that uses the closed-loop controlled ventilatory mode. It uses the decelerating flow waveform and it is based on the optimal or intelligent targeting scheme. The ventilator automatically adjusts its output (tidal volume, respiratory rate, flow time, and inspiratory time) based on the calculation of time constant of the expiratory flow. The ventilator analyzes the flow-volume curve and adjusts the I:E relation and the target rate to keep the target volume within a margin of safety and prevent auto-PEEP, volu-trauma, and baro-trauma [21, 22]. Proportionalassist ventilator is another ventilator mode that calculates the patient's inspiratory effort and provides variable support (inspiratory flow and tidal volume) depending on the amplification setting of the patient's effort (percentage support) to unload the respiratory muscles [23].

\section{Calculation of respiratory mechanics}

The traditional calculation of respiratory mechanics is described using both an inspiratory and expiratory flow that pauses maneuvers in the volume-controlled mode using the square waveform as below. The plateau pressure can be measured by inspiratory hold maneuver or the addition of an inspiratory pause on every breath (Table 1, Equations 1 and 2).

Another way of breath-to-breath automatic calculation of the dynamic respiratory mechanics (compliance, resistance, and auto-PEEP) without the need for any pause maneuvers is termed the least square fitting method, which uses regression analysis of the airway pressure, flow, and volume curves and applies it to the equation of motion (below). Those measurements are available in many new-generation ventilators [24]; however, those calculations might not be accurate in the actively 


\section{FIGURE 6.}

Effect of cycling sensitivity on flow-time curve during pressure support ventilation (A-C) and airway pressure and flow curves versus time in pressure support (D and E). (A) Low sensitivity $5 \%$ causing increased time and failure to trigger; (B) adequate sensitivity of $25 \%$; (C) high sensitivity of $60 \%$ causing short inspiratory time; (D) adequate rise time of 25 milliseconds, note the right angle of airway pressure and high flow of $90 \mathrm{~L} / \mathrm{min}$; and (E) same settings of pressure support except very low inspiratory rise of 200 milliseconds, note the obtuse angle on airway pressure and low flow rate of $50 \mathrm{~L} / \mathrm{min}$. Upper yellow curves are the airway pressures in $\mathrm{cmH}_{2} \mathrm{O}$, lower pink curves are flows in $\mathrm{L} / \mathrm{min}$.
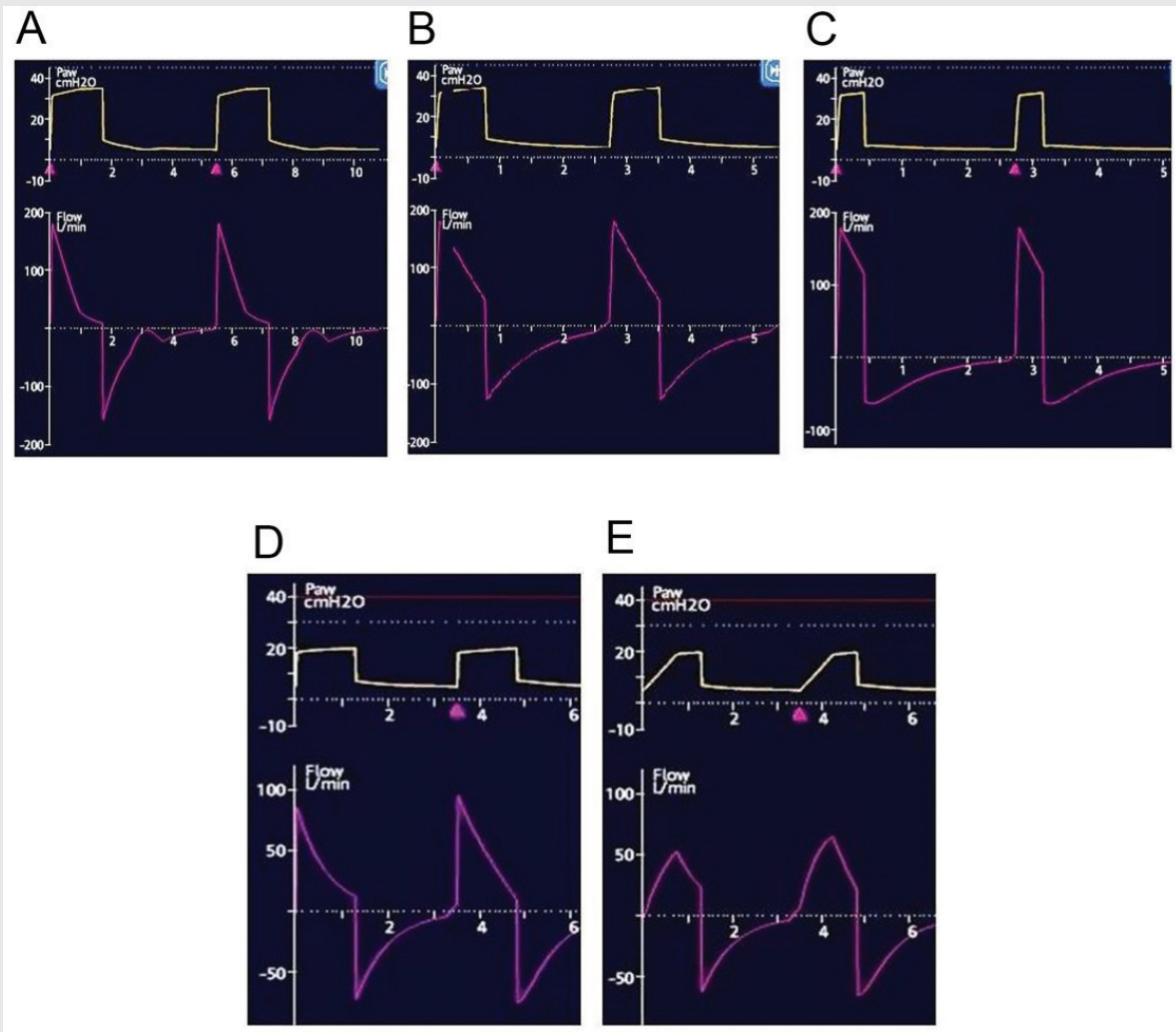

\section{TABLE 1}

\section{Equation of the respiratory mechanics}

\begin{tabular}{|c|c|}
\hline Equation number & Equation \\
\hline $\begin{array}{l}1 \\
2 \\
3\end{array}$ & $\begin{array}{l}\text { Total respiratory system compliance }\left(\mathrm{C}_{\mathrm{RS}} \mathrm{mL} / \mathrm{cmH}_{2} \mathrm{O}\right)=\mathrm{V}_{\mathrm{T}} \text { (exhaled)/Plateau pressure }- \text { Total PEEP (applied + auto-PEEP) } \\
\text { Total airway resistance }\left(\mathrm{R}_{\text {aw }} \mathrm{cmH}_{2} \mathrm{O} / \mathrm{L} / \mathrm{s}\right)=\mathrm{PIP}-\mathrm{Plateau} \text { pressure/Flow in } \mathrm{cmH}_{2} \mathrm{O} / \mathrm{L} / \mathrm{s}[25] \\
\text { Ptotal = Pvent + Pmus }=\mathrm{V}^{\top} / \mathrm{CRS}+\mathrm{Raw} \times \mathrm{V}^{\circ}+\mathrm{PEEP} \text { total }\end{array}$ \\
\hline
\end{tabular}

Note: PTotal, total pressure required to move tidal volume in $\mathrm{cmH}_{2} \mathrm{O}$; Pvent, airway pressure in $\mathrm{cmH}_{2} \mathrm{O} ; \mathrm{Pmus}$, patient's muscle pressure, in cmH $\mathrm{O}_{2}$; $\mathrm{VT}$, tidal volume in $\mathrm{mL}$; CRS, respiratory system compliance in $\mathrm{mL} / \mathrm{cm} \mathrm{H}_{2} \mathrm{O} ; \mathrm{Raw}$, airway resistance in $\mathrm{cm} \mathrm{H} 2 \mathrm{O} / \mathrm{L} / \mathrm{s} ; \mathrm{V}, \mathrm{flow}$ in $\mathrm{L} / \mathrm{s}$; $\mathrm{PEEPi}$, the intrinsic $\mathrm{PEEP}$ in $\mathrm{cm} \mathrm{H}_{2} \mathrm{O}$.

breathing patient compared with the passive patient with no effort given the unknown value of the Pmus (Table 1, Equation 3).

Detection of auto-PEEP/Hyperinflation

Hyperinflation and auto-PEEP can have deleterious effects during mechanical ventilation, including adverse hemodynamic effects, missed trigger, increased WOB, asynchronies, and delayed weaning. This more often occurs in obstructive airway diseases like asthma or COPD; however, it can happen in other conditions, such as ARDS, mucus plugs, and dynamic hyperinflation without airflow obstruction (inadequate expiratory time) $[1,26]$. Higher $V_{T}, R R$, and shorter expiratory time contribute to this phenomenon. Early detection and correction of such a phenomenon are of paramount importance. These may be detected in the expiratory flow-time curve when it does not reach the baseline before the next breath or in the flow-volume curve (Figure 7) when expiratory flow does not reach the baseline.

Quantification of auto-PEEP is usually done via the end-expiratory pause maneuver. Corrections include reducing minute ventilation (tidal volume and respiratory rate) or increasing the expiratory time (by increasing inspiratory flow in VCV modes or reducing the I-time or I:E ratio in the pressure-controlled modes). Increasing applied PEEP to $70 \%-80 \%$ of total PEEP has been suggested for intrinsic expiratory flow obstruction such as COPD [19, 26]. Disconnecting from mechanical ventilation to allow adequate exhalation of the trapped air has been described, though the potential derecruitment of the lung is worrisome, especially in conditions of increased elasticity like ARDS. This maneuver should only be done only in extenuating circumstances [27]. 


\section{FIGURE 7.}

Flow-volume curves in different clinical scenarios (A-C) and flow-pressure loop in different clinical scenarios (D-F). (A-C) Flow in L/min on x-axis, tidal volume in $\mathrm{mL}$ on $\mathrm{y}$-axis. Respiratory cycle going anti clockwise with inspiration marked positive flow and expiration with negative flow. (A) Normal respiratory compliance and resistance, (B) high compliance and resistance (e.g., COPD), (C) low compliance and normal resistance (e.g., ARDS). (D-F) Airway pressure in $\mathrm{cmH}_{2} \mathrm{O}$ on $\mathrm{x}_{-}-\mathrm{axis}$, flow in L/min on y-axis. (D) Normal resistance and compliance, (E) high resistance and normal compliance COPD, (F) low compliance and normal resistance ARDS.
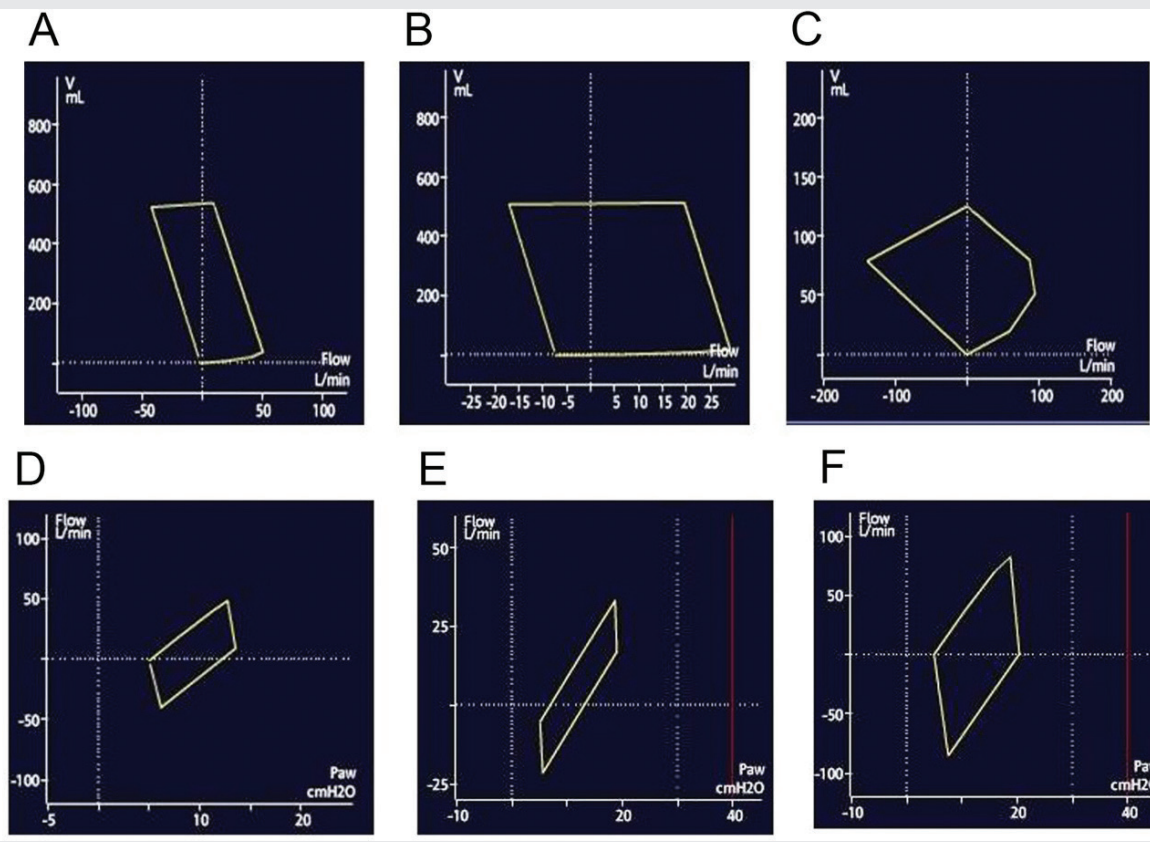

$\mathrm{E}$

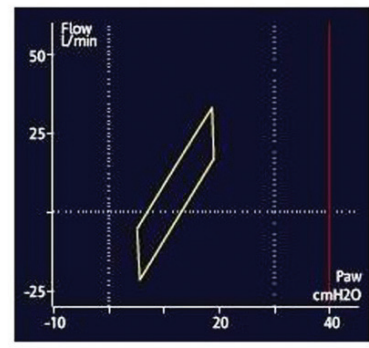

$\mathrm{F}$

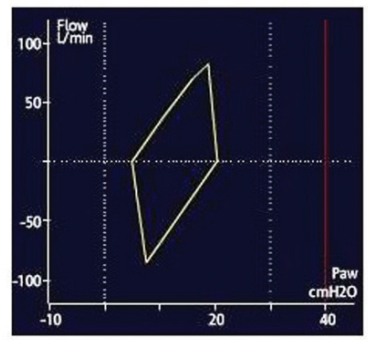

\section{Setting inspiratory time}

As described above, PCV modes use decelerating inspiratory flow ramps, if the inspiratory time is inadequately short, then the resultant $\mathrm{V}_{\mathrm{T}}$ will be lower, which can cause inadequate recruitment and result in hypoxia. If it is higher than optimal, no extra volumes will be gained, which can cause the patient-ventilator asynchrony [1]. Sometimes this strategy is needed, such as in cases of severe hypoxia in ARDS to increase Paw like in cases of inverse ratio ventilation or airway pressure release ventilation (APRV) [1] (Figure 8).

Setting expiratory time

As explained above, inadequate expiratory time can lead to autoPEEP and an appropriate setting of expiratory time is essential. In APRV, the expiratory flow is important in setting the release time also known as time low or T-Low. It is usually set very low in the millisecond to intentionally create auto-PEEP, some research suggested setting the T-Low when the expiratory flow decreases to $50 \%$ $75 \%$ of the PEF [28] (Figure 8).

\section{Rise time}

Once the ventilator is triggered in a PCV mode, Paw increases exponentially to the driving pressure or pressure support level and then stays at the level until the termination of the inspiratory phase (cycling). The "rise time" is the slope until the inspiratory flow reaches the maximum and it should be adjusted to patient comfort (Figure 6). Fast and slow rise times are associated with high and slow flow at the onset of inhalation, respectively, and this should be tailored based on the patient's respiratory drive [19]. For patients with COPD, and restrictive lung disease, faster rise time provided the lowest WOB. In one small study with 15 patients, fast rise time was as effective as increasing the level of pressure support [29].

\section{Flow-volume loop}

The flow-volume loop has been used in the outpatient pulmonary function tests as an objective measure of airway resistance. It differentiates whether the resistance is dynamic or fixed and whether its location is intra- or extra-thoracic. With the advanced computing of mechanical ventilators, they can display the flow-volume loops breath to breath. They provide some useful information, for example auto-PEEP can be identified when the flow does not return to zero before the next breath as described above. Additionally, the expiratory flow decay curve signifying effects of airway resistance. Clinicians can use the flow-volume curve as a tool to evaluate the response to bronchodilator (e.g., increased peak expiratory flow, faster decay of the expiratory curve, and resolution of auto-PEEP) or to assess the need for these treatments in patients with airway obstruction (Figure 7).

In addition, "saw tooth" artifacts on the flow-volume as well as flow-time curve signals the need of airway suctioning for the presence of mucus or water condensation in the circuit or within the large airways.

\section{Flow-pressure loop}

Some ventilators offer a flow versus pressure loop. Though the significance of this curve is not mentioned in the mechanical ventilation literature, it has been described in physics and engineering literature. For gas to flow from the ventilator to the patient during inspiration there should be a pressure gradient between the ventilator and the alveoli also known as the driving pressure.

The relation between pressure and flow was described by the Poiseuille law, it describes the pressure difference between two ends of a pipe that are proportional to the length, dynamic viscosity, and volumetric flow rate while inversely related to the radius of the pipe [30]. 


$$
\Delta \mathrm{p}=8 \mu \mathrm{LQ} / \pi \mathrm{R}^{4}=8 \pi \mu \mathrm{LQ} / \mathrm{A}^{2}
$$

where $\Delta \mathrm{p}$ is pressure difference between the two ends, $\mathrm{L}$ is the length of the pipe, $\mu$ is the dynamic viscosity, $Q$ is the volumetric flow rate, $R$ is the pipe radius, and $\mathrm{A}$ is the cross-section of the pipe.

\section{FIGURE 8.}

Airway pressure and flow curves versus time in APRV. Note the difference in the flow waveform between the mandatory breath as a decelerating waveform (white arrow) and spontaneous breath sinusoidal waveform (yellow arrow). In this example, the peak expiratory flow is $-120 \mathrm{~L} / \mathrm{min}$ (bottom green arrow), while the inspiratory flow starts at $-40 \mathrm{~L} / \mathrm{min}$ (top green arrow) which is $33 \%$ of the PEF. Upper yellow curves are the airway pressures in $\mathrm{CmH}_{2} \mathrm{O}$, lower pink curves are flows in L/min.

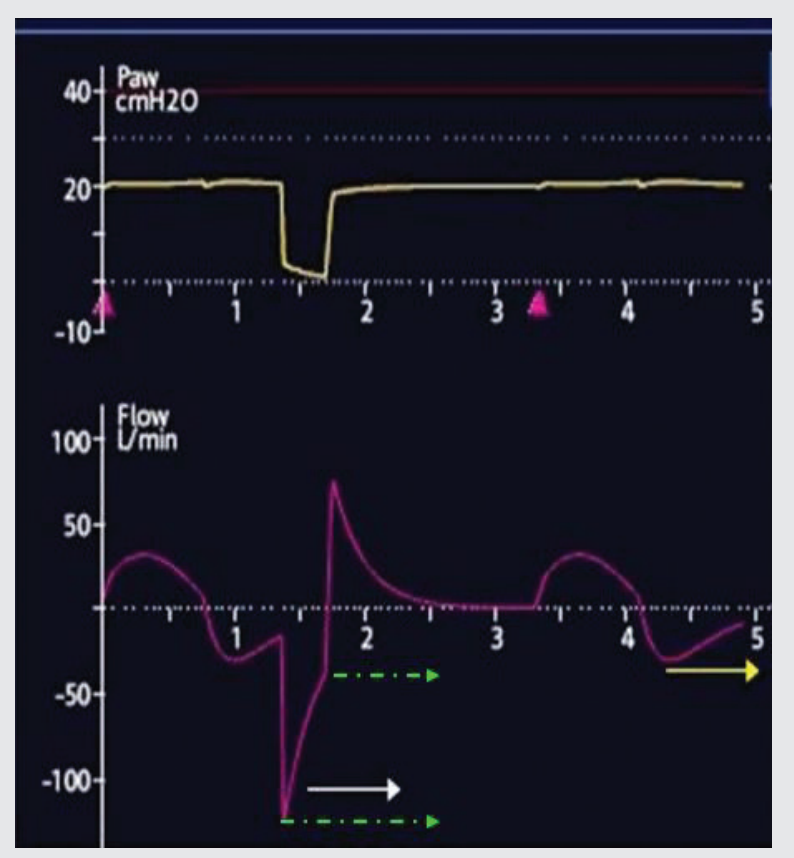

As described above, the airway resistance is the value of the airway pressure drop from the peak to plateau pressures over the flow rate, thus concluding that the flow-pressure loop basically describes airway resistance during inspiration and expiration (Figure 7).

\section{Asynchronies}

Patient-ventilator asynchronies are encountered frequently during mechanical ventilation [31]. There are many kinds and classifications of asynchronies. Most of the asynchronies, unfortunately, go unnoticed, but their existences have been linked to prolonged mechanical ventilation, difficult weaning, reduced patient comfort, increased risk of diaphragmatic damage, and potential increased morbidity and mortality [32]. Reviewing all asynchronies is beyond the scope of this article, we will review some of the asynchronies that can be detected by observing the flow-time curve. Figure 9 summarizes those asynchronies.

Trigger asynchronies usually happen at the beginning of the breath. The patient's effort can be detected as a positive deflection on the flow curve even if still negative [33] (Superscripts 1 and 2 correspond to numbers used in Figure 9.).

A) Delayed triggering occurs when the time between the patient's effort to the delivery of the breath is prolonged. It is characterized by prolonged time from the positive deflection in flow ${ }^{1}$ to the ventilator-delivered breath ${ }^{2}$. It is usually caused by inappropriately high settings of trigger sensitivity, presence of auto-PEEP, weak effort, or low respiratory drive.

B) Missed triggering occurs when the patient's inspiratory effort does not trigger a breath from the ventilator. It is characterized by a positive deflection in flow ${ }^{1}$ and not followed by a breath ${ }^{2}$. Causes are similar to the case of delayed triggering alongside high-pressure support settings, high tidal volumes, and high-set respiratory rate and inspiratory time.

C) Double triggering occurs when the ventilator cycles to exhalation while the patient's continued inspiratory activity can retrigger the ventilator. It is characterized by two assisted breaths without expiration between them or with an expiration interval less than half the mean inspiratory time (two inspiratory peaks). This can be caused by early cycling of the breath, (e.g., high expiratory trigger sensitivity (ETS) in the pressure support modes, high level of pressure support, the very short time constant, or very high respiratory drive) [1]. Cycling asynchronies happen when there is

\section{FIGURE 9.}

Flow asynchronies. Courtesy of Hamilton medical (www.hamilton-medical.com/asynchronies). (A) Delayed triggering. 1: start of patient effort indicated by positive deflection in flow, 2: start of ventilator delivery of breath. Dashed line indicates delay of breath delivery. (B) Ineffective effort. 1: start of patient effort indicated by positive deflection in flow. 2: ineffective effort as no breath. Delivered by the ventilator. Dashed line indicates where the breath should have been delivered. (C) Double triggering. Two mechanical breaths are delivered during one single inspiratory effort. Dashed line indicates where the breath should have been delivered. (D) Auto triggering. 1: patient continued respiratory effort as small bump at the beginning of expiration (after peak expiratory flow). 2: abrupt initial reversal in the expiratory flow. Dashed line shows a normal passive expiratory flow. (E) Early cycling. 1: fast decline in inspiratory flow. 2: slow decrease in flow decline. Dashed line indicates where the cycling should optimally start.

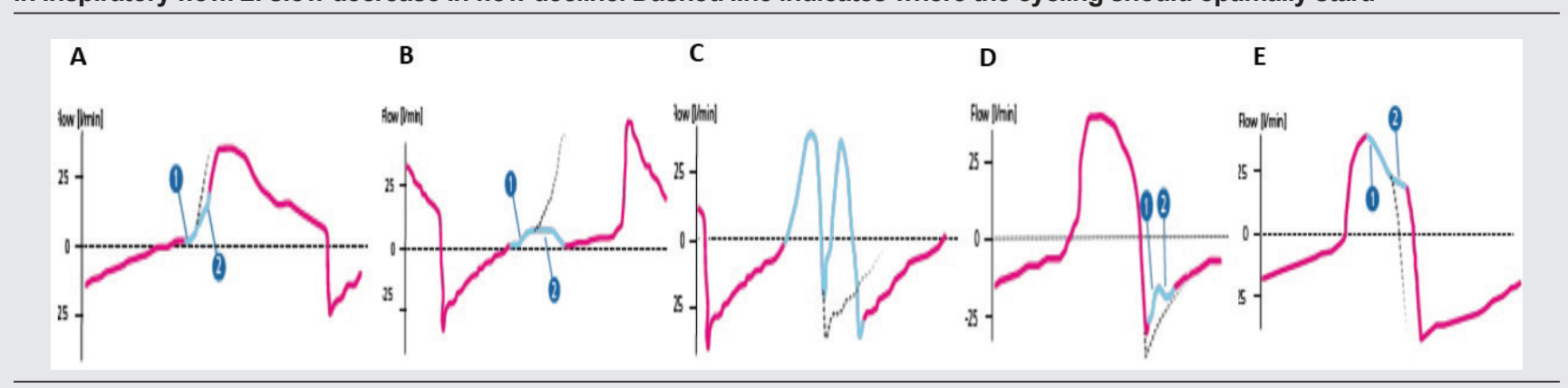


a mismatch between the patient and the ventilator inspiratory and expiratory times.

D) Early cycling occurs when the ventilator cycles from inspiration to expiration despite continued effort of the patient. It is characterized by observing the peak expiratory flow (PEF) which is low, looks doubled and "bumpy," and may cause double triggering. It is usually caused by inappropriately high ETS, low levels of pressures in PSV mode, or short inspiratory time in time cycled mode or high patient inspiratory drive.

E) Delayed cycling occurs when the inspiratory flow time is longer than the patient's inspiratory muscle effort. It is characterized by observing a steep decline in the inspiratory flow followed by a less steep decline before it cycles to expiration. Causes are the opposite of early cycling including low ETS, high inspiratory time, high tidal volume, high-pressure support values, and low flow rate [1].

\section{Future research}

The effects of the patient's efforts during the mechanical ventilator liberation or a spontaneous breathing trial have been studied. Multiple parameters and indices have been developed in attempts to predict the success or the failure of the weaning trial [34]. Unfortunately, most of these parameters and indices lack good predictive values. In our simulator work testing noninvasive ways of measuring Pmus, we noticed the effects of Pmus on the inspiratory flow-time curve with increasing concavity and mean flow with a higher Pmus. We are currently conducting a study to test this relationship between muscle pressures and mean inspiratory flow to evaluate if it can be used as an indicator of success versus failure of a weaning trial. This concept needs to be proven and studied further before any clinical application or recommendations.

\section{Resistance calculation}

As described above, the effects of airway resistance could conceptually be calculated dynamically from the flow-pressure curves during inspiration and expiration without any pause maneuvers. Similarly, this concept needs to be proven and studied further before any clinical application.

\section{CONCLUSION}

Flow waveforms shapes and patterns are very beneficial for the management of patients undergoing mechanical ventilatory support. Attention to those waveforms can potentially improve patient outcomes. Clinicians should be familiar with this information and how to act upon them. Online ventilator simulators are available and good sources to practice [33].

\section{Contributors}

$\mathrm{NH}, \mathrm{RS}, \mathrm{ED}$ all contributed to the design of the work, collecting previous studies, and drafting and commenting on the paper, approved the final version of this paper. ED contributed to editing the figures.

\section{Funding}

This study did not receive any specific grant from funding agencies in the public, commercial, or not-for-profit sectors.

\section{Competing interests}

All authors have completed the ICMJE uniform disclosure form at www. icmje.org/coi_disclosure.pdf and declare: no financial relationships with any organizations that might have an interest in the submitted work in the previous 3 years; no other relationships or activities that could appear to have influenced the submitted work.

\section{REFERENCES}

1. Tobin MJ. Principles and practice of mechanical ventilation, third edition. McGraw Hill Professional, New York; 2012.

2. Cannon ML, Cornell J, Tripp-Hamel DS, et al. Tidal volumes for ventilated infants should be determined with a pneumotachometer placed at the endotracheal tube. Am J Respir Crit Care Med 2000;162(6):2109-12. doi: $10.1164 /$ ajrccm.162.6.9906112

3. Iotti GA, Braschi, A. Measurements of respiratory mechanics during mechanical ventilation, Hamilton Medical Scientific Library, Rhazuns, Switzerland (1999). hamiltonmedical.nl.
4. Silvestri S. The influence of flow rate on breathing circuit compliance and tidal volume delivered to patients in mechanical ventilation. Physiol Meas 2005;27(1):23-33. doi: 10.1088/0967-3334/27/1/003

5. Daoud EG, Farag HL, Chatburn RL. Airway pressure release ventilation: What do we know? Respir Care 2012;57(2):282-92. doi: 10.4187/ respcare. 01238

6. Bergman NA. Intrapulmonary gas trapping during mechanical ventilation at rapid frequencies. Anesthesiology 1972;37(6):626-33. doi: 10.1097/00000542-197212000-00011

7. Lucangelo U, Bernabé F, Blanch L. Respiratory mechanics derived from signals in the ventilator circuit. Respir Care 2005;50(1):55-65: discussion 65-7.

8. Mughal MM, Culver DA, Minai OA, Arroliga AC. Auto-positive endexpiratory pressure: Mechanisms and treatment. Cleve Clin J Med 2005;72(9):801-9. doi: 10.3949/ccjm.72.9.801

9. Marini JJ. Dynamic hyperinflation and auto-positive end-expiratory pressure. Am J Respir Crit Care Med 2011;184(7):756-62. doi: 10.1164/ rccm.201102-0226PP

10. Detajin Junhasavasdikul MD, Irene Telias MD, Domenico Luca Grieco $\mathrm{MD}$, et al. Expiratory flow limitation during mechanical ventilation. Chest 2018;154(4):1-38. doi: 10.1016/j.chest.2018.01.046

11.Marini JJ. Point: Is pressure assist-control preferred over volume assistcontrol mode for lung protective ventilation in patients with ARDS? Yes. Chest 2011;140(2):286-90. doi: 10.1378/chest.11-1060

12.Rittayamai N, Katsios CM, Beloncle F, Friedrich JO, Mancebo J, Brochard L. Pressure-controlled vs volume-controlled ventilation in acute respiratory failure. Chest 2015;148(2):340-55. doi: 10.1378/ chest. 14-3169

13. Yang SC, Yang SP. Effects of inspiratory flow waveforms on lung mechanics, gas exchange, and respiratory metabolism in COPD patients during mechanical ventilation*. Chest 2002;122(6):2096-104. doi: 10.1378/ chest.122.6.2096

14. Al-Saady N, Bennett ED. Decelerating inspiratory flow waveform improves lung mechanics and gas exchange in patients on intermittent positive-pressure ventilation. Intensive Care Med 1985;11(2):68-75. doi: 10.1007/BF00254777

15. Lucangelo U, Bernabé F, Blanch L. Lung mechanics at the bedside: Make it simple. Curr Opin Crit Care 2007;13(1):64-72. doi: 10.1097/MCC. Ob013e32801162df

16. Kondili E, Alexopoulou C, Xirouchaki N, Vaporidi K, Georgopoulos D. Estimation of inspiratory muscle pressure in critically ill patients. Intensive Care Med 2010;36(4):648-55. doi: 10.1007/s00134-010-1753-4

17. Fujita Y, Fujino Y, Uchiyama A, Mashimo T, Nishimura M. High peak inspiratory flow can aggravate ventilator-induced lung injury in rabbits. Med Sci Monit 2007;13(4):BR95-100.

18. Kondili E, Prinianakis G, Georgopoulos D. Patient-ventilator interaction. Br J Anaesth 2003;91(1):106-19. doi: 10.1093/bja/aeg129

19. Hess DR. Ventilator waveforms and the physiology of pressure support ventilation. Respir Care 2005;50(2):166-86: discussion 183-6.

20. Ranieri VM, Mascia L, Petruzzelli V, Bruno F, Brienza A, Guiliani R. Inspiratory effort and measurement of dynamic intrinsic PEEP in COPD patients: Effects of ventilator triggering systems. Intensive Care Med 1995;21(11):896-903. doi: 10.1007/BF01712330

21. Fernández J, Miguelena D, Mulett H, Godoy J, Martinón-Torres F. Adaptive support ventilation: State of the art review. Indian J Crit Care Med 2013;17(1):16-22. doi: 10.4103/0972-5229.112149

22. Chatburn RL, El-Khatib M, Mireles-Cabodevila E. A taxonomy for mechanical ventilation: 10 fundamental maxims. Respir Care 2014;59(11):1747-63. doi: 10.4187/respcare.03057

23. Ambrosino N. Proportional assist ventilation (PAV): A significant advance or a futile struggle between logic and practice? Thorax 2002;57(3):272-6. doi: 10.1136/thorax.57.3.272

24. Daoud EG, Katigbak R, Ottochian M. Accuracy of the ventilator automated displayed respiratory mechanics in passive and active breathing conditions: A bench study. Respir Care 2019, 64(12):1555-1560. doi: 10.4187/ respcare.06422

25. Dhand R. Ventilator graphics and respiratory mechanics in the patient with obstructive lung disease. Respir Care 2005;50(2):246-61: discussion 259-61.

26. Mora Carpio AL, Mora JI. Positive End-Expiratory Pressure (PEEP). 2019.

27. Blanch L, Bernabé F, Lucangelo U. Measurement of air trapping, intrinsic positive end-expiratory pressure, and dynamic hyperinflation in mechanically ventilated patients. Respir Care 2005;50(1):110-23: discussion 123-4. 
28. Habashi NM. Other approaches to open-lung ventilation: Airway pressure release ventilation. Critical Care Medicine 2005;33(Supplement):S228-40. doi: 10.1097/01.CCM.0000155920.11893.37

29. Uchiyama A, Imanaka H, Taenaka N. Relationship between work of breathing provided by a ventilator and patients' inspiratory drive during pressure support ventilation; effects of inspiratory rise time. Anaesth Intensive Care 2001;29(4):349-58. doi: 10.1177/0310057X0102900404

30. Bates JHT. Systems physiology of the airways in health and obstructive pulmonary disease. Wiley Interdiscip Rev Syst Biol Med 2016;8(5): 423-37. doi: $10.1002 /$ wsbm. 1347

31. The Asynchronies in the Intensive Care Unit (ASYNICU) Group, de Haro C, Ochagavia A, et al. Patient-ventilator asynchronies during mechanical ventilation: Current knowledge and research priorities. Intensive Care Med Exp 2019;7(S1):43. doi: 10.1186/s40635-019. 0234-5

32. Blanch L, Villagra A, Sales B, et al. Asynchronies during mechanical ventilation are associated with mortality. Intensive Care Med 2015;41(4):63341. doi: 10.1007/s00134-015-3692-6

33. Sassoon CSH, Foster GT. Patient-ventilator asynchrony. Curr Opin Crit Care 2001;7(1):28. doi: 10.1097/00075198-200102000-00005

34. Doorduin J, Roesthuis LH, Jansen D, van der Hoeven JG, van Hees HWH, Heunks LMA. Respiratory muscle effort during expiration in successful and failed weaning from mechanical ventilation. Anesthesiology 2018;129(3):490-501. doi: 10.1097/ALN.0000000000002256 